\title{
Comprehensive molecular profiling of intrahepatic cholangiocarcinoma in the Chinese population and therapeutic experience
}

Longrong Wang ${ }^{1}$, Hongxu Zhu², Yiming Zhao ${ }^{1}$, Qi Pan ${ }^{1}$, Anrong Mao ${ }^{1}$, Weiping Zhu', Ning Zhang ${ }^{1}$, Zhenhai Lin ${ }^{1}$, Jiamin Zhou' ${ }^{1}$, Yilin Wang ${ }^{1}$, Yongfa Zhang ${ }^{1}$, Miao Wang ${ }^{1}$, Yun Feng ${ }^{1}$, Xigan $\mathrm{He}^{2}$, Weiqi Xu and Lu Wang ${ }^{1 *}$ (D)

\begin{abstract}
Background: The genomic alterations of intrahepatic cholangiocarcinoma (ICC) in the Chinese population have not been fully revealed. Molecular profiling may provide a reference for clinical management, especially targeted therapy.

Methods: A retrospective study was conducted in $122 \mathrm{ICC}$ patients. All patients' samples underwent next-generation sequencing (NGS), which analyzed 417 genes. The genetic characteristics, clinical management and therapeutic responses were analyzed.

Results: The most commonly mutated genes were TP53 (34\%), KRAS (25\%) and ARID1A (17\%). Targeted agents were used referring to molecular profiling, in combination with chemotherapy. Twenty-two patients with wild-type KRAS/ NRAS/BRAF were treated with cetuximab. The disease control and response rates were $78 \%$ and $47 \%$, respectively, which were higher than those achieved with chemotherapy alone $(72 \%$ and $11 \%, P=0.16)$. Fifty-four patients underwent anti-VEGF treatment with bevacizumab. The disease control and response rates were $85 \%$ and $60 \%$, respectively. Better therapeutic efficiency ( $P=0.001)$ and longer progression-free survival $(P F S)$ were observed in the bevacizumabtreated group compared to chemotherapy alone group (15.4 and 6.7 months, respectively; $P=0.04$ ). The PFS of ten patients who underwent hepatectomy after combined treatment with chemotherapy and bevacizumab was longer than that of 139 patients who underwent surgical treatment (28.9 vs 18.0 months, $P=0.03$ ). Two patients (1.6\%) had signatures of microsatellite instability (MSI-H), and both benefited from immunotherapy.
\end{abstract}

Conclusions: This study provides an overview of genetic alterations in Chinese ICC patients and indicates the potential clinical implications for NGS-based personalized therapies.

Keywords: Intrahepatic cholangiocarcinoma, Next-generation sequencing, Molecular profiling, Target therapy, Immune therapy

*Correspondence: wang.lu99@hotmail.com

1 Liver Surgery Department, Shanghai Cancer Center, Fudan University, No. 270 Dongan Rd., Shanghai 200032, People's Republic of China Full list of author information is available at the end of the article

\section{Background}

The incidence of liver cancer ranks 4th among the Chinese population according to data published by the National Central Cancer Registry of China (NCCR) [1]. Intrahepatic cholangiocarcinoma (ICC) is the second most common type and accounts for $10-20 \%$ of all

(C) The Author(s) 2020. This article is licensed under a Creative Commons Attribution 4.0 International License, which permits use, sharing, adaptation, distribution and reproduction in any medium or format, as long as you give appropriate credit to the original author(s) and the source, provide a link to the Creative Commons licence, and indicate if changes were made. The images or other third party material in this article are included in the article's Creative Commons licence, unless indicated otherwise in a credit line to the material. If material is not included in the article's Creative Commons licence and your intended use is not permitted by statutory regulation or exceeds the permitted use, you will need to obtain permission directly from the copyright holder. To view a copy of this licence, visit http://creativecommons.org/licenses/by/4.0/. The Creative Commons Public Domain Dedication waiver (http://creativecommons.org/publicdomain/zero/1.0/) applies to the data made available in this article, unless otherwise stated in a credit line to the data. 
primary liver cancers $[2,3]$. The increasing incidence rate and aggressive clinical course of ICC contribute to its high mortality [4]. Surgical resection remains the mainstay of potentially curative treatment for patients with early-stage disease, but few treatment options are available for the majority of patients with advanced-stage or unresectable disease. The combination of gemcitabine and cisplatin is still the standard of care for patients with advanced and metastatic disease, and no standard targeted therapy has been proven in clinical trials [5-7]. Pembrolizumab and pemigatinib have been currently approved to treat $10 \%$ of patients with specific genetic characteristics, while precise treatments are urgently needed to improve the survival of the remaining $90 \%$ of patients with advanced disease.

Next-generation sequencing (NGS) is an ideal tool to categorize patients with ICC based on molecular profiles [8], and few large-scale sequencing studies have focused on the genomic characteristics of ICC in the Chinese population. The molecular phenotypes of ICC have not yet been revealed and represent a rational personalized therapeutic approach.

In this study, NGS was performed on 122 Chinese ICC patient samples to elucidate the molecular profiles, and target or immune agents were administered based on the genetic characteristics.

\section{Methods}

\section{Patients}

Patients were identified over a 4-year period starting in April 2015 and were deemed eligible for the study if they had a confirmed histological diagnosis of ICC. Written informed consent for tumor profiling was obtained from each patient upon their first admission to Fudan University Shanghai Cancer Center (FUSCC). The study protocol was approved by the FUSCC ethics committee (No. 218-1611 and No. 050432-4-1911D).

The clinical data and NGS results for 122 patients with ICC were available at the time of analysis. Overall survival (OS) and progression-free survival (PFS) rates were collected.

Survival data of 139 patients accepted curative surgery for ICC in the same center was used.

\section{Sample collection and preparation}

Previously collected fresh tissue and blood samples were used in this study. The tissues were obtained through laparoscopic surgery or core needle biopsy. The fresh tissue was soaked in 5 times the volume of $4 \%$ formaldehyde solution within 30 min. A wax block was made within $24 \mathrm{~h}$ after soaking the tissue, and it was sent to the pathologist for diagnosis and review. The specimens were sent to the laboratory for NGS detection within $48 \mathrm{~h}$ at
$4-8{ }^{\circ} \mathrm{C}$. Twenty milliliters of peripheral blood was drawn and sent to the laboratory within $48 \mathrm{~h}$ at $15-35^{\circ} \mathrm{C}$.

Tissue samples with an estimated tumor purity $<10 \%$ based on histopathological assessment were deemed insufficient for sequencing. The standard amount of DNA input was $250 \mathrm{ng}$, and the minimum input was $50 \mathrm{ng}$ in cases for which the DNA quality was limited. Matched germline DNA from prospectively collected blood samples was analyzed for all patients.

\section{Tissue and plasma DNA isolation and purification}

Genomic DNA (gDNA) was extracted from formalinfixed, paraffin-embedded (FFPE) samples using the GeneRead DNA FFPE Kit (Qiagen, USA), and gDNA was extracted from the white blood cell samples using the DNA Blood Midi/Mini kit (Qiagen, USA). The quality of purified DNA was assayed by gel electrophoresis and quantified by the Qubit ${ }^{\circledR} 4.0$ fluorometer (Life Technologies, USA).

\section{Library construction and bioinformatics analysis}

Purified gDNA was first fragmented into DNA pieces approximately 200-300 bp in size using an enzymatic method (5X WGS Fragmentation Mix, Qiagen, USA). After end repair, tailing and T-adaptor ligation by polymerase chain reaction (PCR) was used to generate a prelibrary, and the products were then subjected to exon capture. Captured fragments were subsequently purified and hybridized by a 417-gene panel (Additional file 1: Table S1). FASTP [9] was used to trim adapters and remove low-quality sequences to obtain clean reads, which were aligned to the Ensemble GRCh37/ hg19 reference genome by BWA [10]. PCR duplicates were processed by GenCore [11], and consensus reads were generated. SAMtools [12] was utilized for the detection of single-nucleotide variations (SNVs), insertions and deletions, and Human Genome Variation Society (HGVS) variant descriptions were annotated by ANNOVAR [13] software. After annotation, SNVs with a PopFreqMax $>0.05$ were excluded, and nonsynonymous SNVs with a variant allele frequency $(\mathrm{VAF})>0.5 \%$ or a VAF $>0.1 \%$ in cancer hotspots collected from the patient database were retained for further analysis.

The microsatellite instability (MSI) statuses of all tissue samples were determined, and this score was used to classify the samples into three groups, MSI-high, $\geq 2$ unstable microsatellite loci; MSI-low, only 1 instable locus; and microsatellite stable (MSS), no locus instability. The MSI-high results were further confirmed by PCR validation.

The tumor mutational burden (TMB) was estimated by somatic nonsynonymous mutations per megabase of the panel sequences examined. 
Pathway enrichment was conducted in KEGG website (https://www.kegg.jp).

\section{Immunohistochemical analysis of PD-L1}

The tissue was placed in a $4 \%$ paraformaldehyde solution for $12 \mathrm{~h}$. Then, the tissues were dehydrated with $75 \%$ and $95 \%$ absolute ethanol each for $1.5 \mathrm{~h}$ at $60-70{ }^{\circ} \mathrm{C}$. Next, the tissues were soaked in $40{ }^{\circ} \mathrm{C}$ dichloromethane for $4 \mathrm{~h}$ and then rehydrated with absolute ethanol, $95 \%$ ethanol, $75 \%$ ethanol and distilled water for $1 \mathrm{~h}, 0.5 \mathrm{~h}, 1 \mathrm{~h}$ and $1 \mathrm{~h}$, respectively, at $60-70{ }^{\circ} \mathrm{C}$. The samples were then embedded in paraffin wax, and ultrathin Sects. $(5 \mu \mathrm{m})$ were cut using an ultramicrotome (Lecia RM2126RT, Germany), mounted on glass slides, and stained with hematoxylin and eosin for analyzing tissue structures using an upright fluorescence microscope (Eclipse TE2000-S, Nikon, Japan).

We performed immunohistochemical studies to evaluate programmed death ligand 1 (PD-L1) expression on tumor cells (TCs) and immune cells (ICs) using the Ventana SP263 assay with the Ventana BenchMark GX system (Roche/Ventana Medical Systems, Tucson, USA) according to the recommended protocol. A rabbit monoclonal anti-human PD-L1 antibody (clone SP263, Roche/ Ventana) was used. The slides were immersed in acetone ( $3 \mathrm{~min})$ and xylene $(10 \mathrm{~min})$ to remove the coverslip; the sections were then rehydrated with alcohol in decreasing concentrations and immersed in distilled water. Antigen retrieval was performed with Cell Conditioner 1 for 64 min against SP263. The sections were then incubated with the specific primary antibody for 16 min against SP263. Subsequently, the sections were treated with the OptiView HQ Linker for 8 min and the OptiView HRP Multimer for $8 \mathrm{~min}$. Finally, counterstaining was performed with Mayer's hematoxylin and Scott's tap water bluing reagent. The evaluation of the stained tissue sections was performed by two investigators who had no knowledge of the patients' clinical status. Cases with discrepancies were jointly re-evaluated until a consensus was reached. PD-L1 expression was calculated as the percentage of membrane staining on TCs or ICs in the overall area of the tumor, regardless of intensity.

\section{Clinical management}

Gemcitabine-based chemotherapy was used as the firstline chemotherapeutic treatment in this study, and combinatory strategies included cisplatin, oxaliplatin and capecitabine. Some patients accepted additional target/ immune therapies (detailed in Additional file 2: Table S2). Patients refused the suggested target/immune therapies would accept chemotherapy alone. The Common Terminology Criteria for Adverse Events (CTCAE) 4.0 criteria were used to evaluate adverse events.
Follow-up was conducted every 8 weeks at the lowest frequency. Enhanced abdominal CT/MR scans and serum CA19-9 levels were examined, and Response Evaluation Criteria in Solid Tumors (RECIST) 1.1 criteria were used to evaluate therapeutic efficacy.

\section{Statistics analysis}

To evaluate the association of clinical characteristics or genes, Fisher's exact test was performed. Odds ratios and false discovery rate (FDR)-corrected $\mathrm{P}$ values were also calculated. PFS was calculated using the Kaplan-Meier method, and the Chi square test was used to compare therapeutic efficiencies between patients treated with different strategies and between those with different genetic alterations. The PFS rate was calculated as the time from the treatment start date to the date of progression or death. For patients who underwent surgical procedures, recurrence after curative resection or progression after palliative surgery was considered progression. Patients alive and without progression were censored to the date of the last follow-up.

\section{Results}

Samples from 122 individual patients with ICC were analyzed. Clinical characters are summarized in Table 1.

A total of 692 genetic alterations were identified among 121 of the 122 samples, while no somatic genetic alterations were identified in the remaining sample. The median number of mutations per sample was 5 , and the most commonly mutated genes were TP53 (34\%), KRAS (25\%), and ARID1A (17\%). Among multiple genes, potentially oncogenic focal copy number variations were noted, including ADAM29 (5\%) and CDKN2A (5\%) deletions and ERBB2 (8\%), CDK12 (4\%), FAM135B (4\%), FRS2 (4\%), and MDM2 (4\%) amplifications. Rearrangements were mostly noted in BCL2L11 (7\%, Fig. 1 and Table 2). Potentially actionable alterations were enriched in FoxO signaling pathway, PI3K-Akt signaling pathway, platinum drug resistance, EGFR tyrosine kinase inhibitor resistance, ErbB signaling pathway, and MAPK signaling pathway (Fig. 2).

Two patient samples (1.6\%) had MSI-H signatures, and the immunohistochemical results for PD-L1 were negative in patient sample A and positive (1\%) in patient sample B (Fig. 3). The total immunohistologically-positive PD-L1 rate in tumor tissues was $16 \%$, and the median expression rate in a single tumor was $5 \%$ (range 1-25\%). The TMBs for patients $A$ and $B$ were 42 and 48 mutations/Mb, respectively. Patient A was a 57-year-old male with a history of hepatitis B virus (HBV) infection, and he was diagnosed with local advanced left lobe cholangiocarcinoma with intrahepatic metastasis, vessel invasion and lymph node metastasis. The patient was treated 
Table 1 Clinical characteristics ( $n=122$ patients)

\begin{tabular}{|c|c|}
\hline Clinical Characteristics & Number (\%) \\
\hline \multicolumn{2}{|l|}{ Sex } \\
\hline Male & $72(59)$ \\
\hline Female & $50(41)$ \\
\hline \multicolumn{2}{|l|}{ Age } \\
\hline Median (range) & $61(33-89)$ \\
\hline \multicolumn{2}{|l|}{ Metastasis } \\
\hline Lymph node & $61(50)$ \\
\hline Intrahepatic & $46(38)$ \\
\hline Lung & $30(25)$ \\
\hline Abdomen/peritoneum & $26(21)$ \\
\hline Bone & $23(19)$ \\
\hline \multicolumn{2}{|l|}{ Invasion } \\
\hline Diaphragm & $16(13)$ \\
\hline Gallbladder & $6(5)$ \\
\hline Adrenal gland & $4(3)$ \\
\hline Colon & $2(2)$ \\
\hline Duodenum & $2(2)$ \\
\hline Stomach & $1(1)$ \\
\hline \multicolumn{2}{|l|}{ Differentiation } \\
\hline Poor & $20(16)$ \\
\hline Moderate & $80(66)$ \\
\hline High & $22(18)$ \\
\hline \multicolumn{2}{|l|}{ Biopsy } \\
\hline Laparoscopic & $31(25)$ \\
\hline Percutaneous & $91(75)$ \\
\hline \multicolumn{2}{|l|}{ Sample analyzed } \\
\hline Tissue & $111(91)$ \\
\hline Blood & $11(9)$ \\
\hline
\end{tabular}

with pembrolizumab for 8 months, and he accepted stereotactic body radiation therapy after the 8th treatment cycle. During radiation therapy, the patient was diagnosed with transient hyponatremia and accepted supportive treatment. The patient exhibited a partial response (Additional file 3: Figure S1) and was alive as of the analysis date. Patient B was a 46-year-old male with a history of HBV infection, and he was diagnosed with local advanced left lobe massive cholangiocarcinoma and lymph node metastasis. After 4 cycles of pembrolizumab treatment, the tumor was deemed stable and shrank by $7 \%$. A left hemihepatectomy without lymphadenectomy was performed at 4 weeks after the 4th treatment cycle, and the 5th treatment cycle began at 4 weeks after the surgery. The patient was alive without tumor metastasis as of the analysis date.

ERBB2 alterations were found in 13 (11\%) patients, and 10 of the 13 patients exhibited ERBB2 gene amplification. Eight patients with ERBB2 amplification received anti-HER2 therapy combined with basic chemotherapy, and the application of trastuzumab achieved a disease control rate of $7 / 8$. Specifically, the stable disease rate was $1 / 8$, the partial response rate was $6 / 8$, and the complete response rate was $0 / 8$. One patient exhibited grade 3 neutropenia, and two patients with partial responses underwent exploratory laparotomy after systemic treatment. The tumor from one patient remained unresectable after surgical exploration, and the other patient accepted radical hemihepatectomy treatment. The patient had not experienced recurrence as of the analysis date. The median PFS time for trastuzumab-treated patients was 7.3 (1-27) months.

BRCA1/2 mutations were detected in 16 (13\%) patients, and most of these mutations were somatic (14 patients, $11 \%)$. One patient with a BRCA2 germline mutation had previously underwent mastectomy for breast cancer before the diagnosis of ICC. Four patients accepted poly ADP-ribose polymerase (PARP) inhibitor (olaparib) treatment combined with chemotherapy. Two patients achieved stable disease, and two patients responded partially. Herein, no cases of complete response or disease progression and no grade 3-4 toxic effects were observed.

KRAS/NRAS/BRAF alterations occurred in $36(30 \%)$ patients. Among the 86 patients with no mutations, 22 accepted cetuximab treatment combined with chemotherapy. The disease control and response rates were $78 \%$ and $47 \%$, respectively, which were higher than those achieved with chemotherapy alone (72\% and $11 \%)$; the differences were not statistically significant $(P=0.16)$, and no complete response was observed. Patients in the cetuximab group had a longer PFS time than those in the chemotherapy group (9.0 vs 6.7 months), but the difference was not statistically significant $(\mathrm{P}=0.51$, Additional file 4: Figure S2). Grade 3 toxic effects included rashes $(n=5)$ and neutropenia $(n=2)$, and no grade 4 toxic effects were observed.

One patient acquired an NRAS mutation in the 5th month of cetuximab treatment, which was diagnosed via imaging and further confirmed by NGS (Additional file 5 : Figure S3).

Fifty-four patients received anti-VEGF treatment (bevacizumab) combined with chemotherapy, and the disease control and response rates were $85 \%$ and $60 \%$, respectively. KRAS/NRAS/BRAF were wild-type in $33 / 54$ patients and mutated in $21 / 54$ patients. Two (4\%) patients had complete responses, and $30(56 \%)$ patients had partial responses. At 6 weeks after treatment, one patient had a stroke, which was diagnosed as a grade 3 adverse event; VEGFA amplification was detected in this patient's specimen. After 4 weeks of treatment, one patient exhibited acute kidney failure, which was diagnosed as a grade 5 adverse event. The clinical features of 


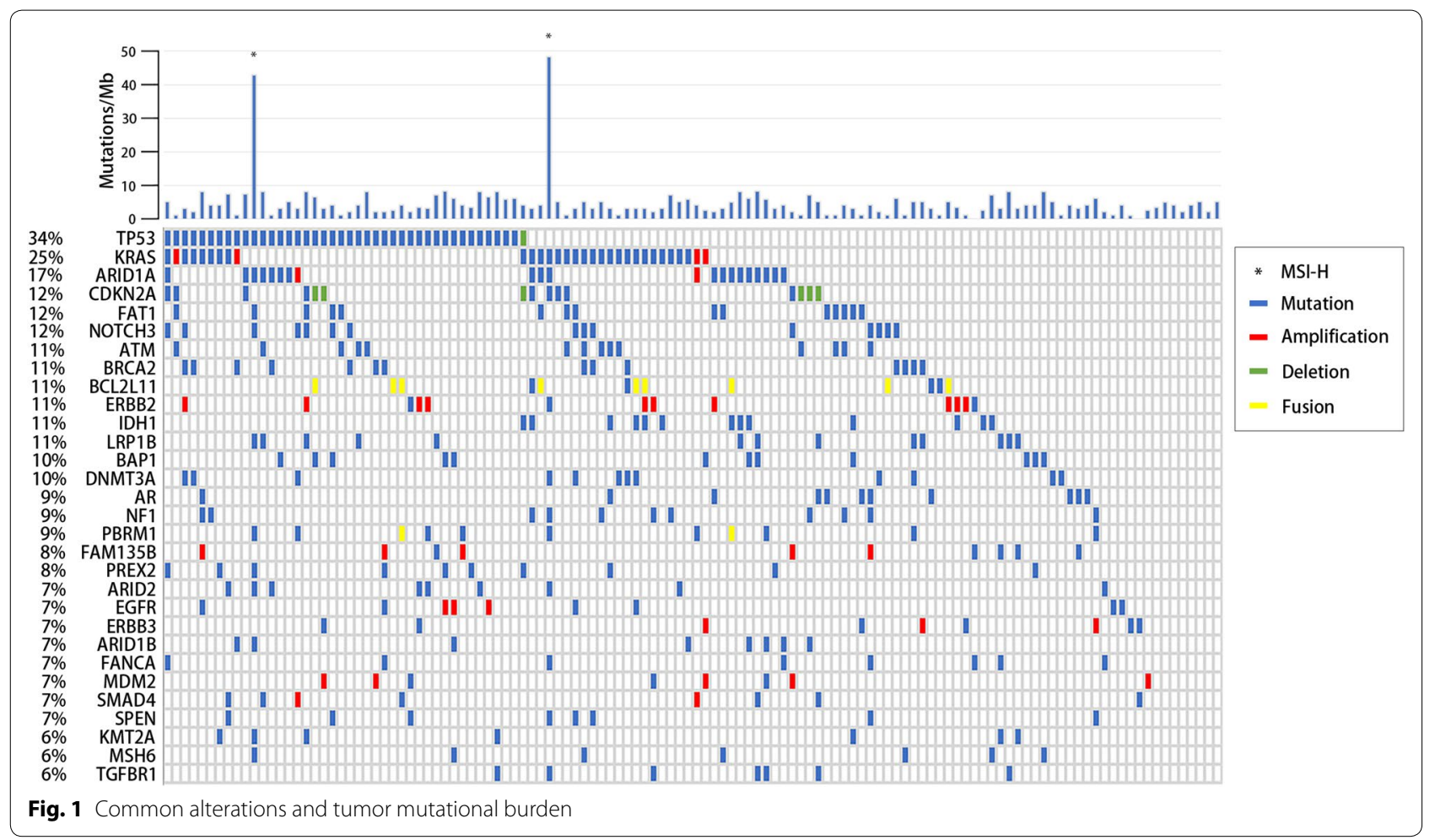

Table 2 Commonly amplified/deleted/rearranged genes

\begin{tabular}{lc}
\hline Gene & Number (\%) \\
\hline Amplification & $10(8)$ \\
ERBB2 & $5(4)$ \\
CDK12/FAM135B/FRS2/MDM2 & $4(3)$ \\
CCNE1/KRAS & \\
Deletion & $6(5)$ \\
ADAM29/CDKN2A & $2(2)$ \\
NTRK1/NTRK3 & $1(1)$ \\
NRG3/TP53/MLH1/SMARCA2 & \\
Rearrangement & $9(7)$ \\
BCL2L11 & $2(2)$ \\
PBRM1 & $1(1)$ \\
ALK/FGFR3(-TACC3) &
\end{tabular}

the patient indicated tumor lysis syndrome (Additional file 6: Figure S4). Other grade 3 toxic effects included neutropenia $(n=2)$ and hypertension $(n=1)$. Patients in the bevacizumab group had a better therapeutic effect $(\mathrm{P}=0.001)$ and a longer PFS time (15.4 vs 6.7 months, $\mathrm{P}=0.04$ ) than those in the chemotherapy group (Fig. 4). After systemic treatment, nine (17\%) patients with partial responses and one (2\%) patient with a complete response underwent exploratory laparotomy followed by hepatectomy. Pathological diagnosis revealed tumor activity in the patient with a clinically complete response. We compared the PFS time of the 10 patients with 139 patients accepted radical hepatectomy treatment in our center. Patients accepted pre-operation systemic treatment acquired a longer PFS time compare to patients accepted surgery treatments along (28.9 vs 18.0 months, $\mathrm{P}=0.03$; Fig. 5). The therapeutic regimens used based on genetic alterations were summarized in Table 3.

Furthermore, overall patients accepted target or immune therapy agencies $(n=90)$ had a longer PFS than those in the chemotherapy group $(n=18 ; 19.3$ vs 6.7 months). There was an obvious tendency, but the difference was not statistically significant $(P=0.053$, Fig. 6$)$.

\section{Discussion}

HBV, hepatitis $\mathrm{C}$ virus (HCV) and chronic cholangitis contribute to the majority of the 70,000 ICC cases emerging in China [1], revealing the heterogeneity of Chinese cases with those of Westerners and South East Asians. Multiple studies have investigated the molecular profiles of cholangiocarcinoma in Western countries, but genetic information for Chinese patients with ICC is lacking. In this study, 417 cancer-associated genes from 122 ICC patient samples were subjected to NGS, and a genetic profile was obtained. TP53, KRAS and ARID1A were 


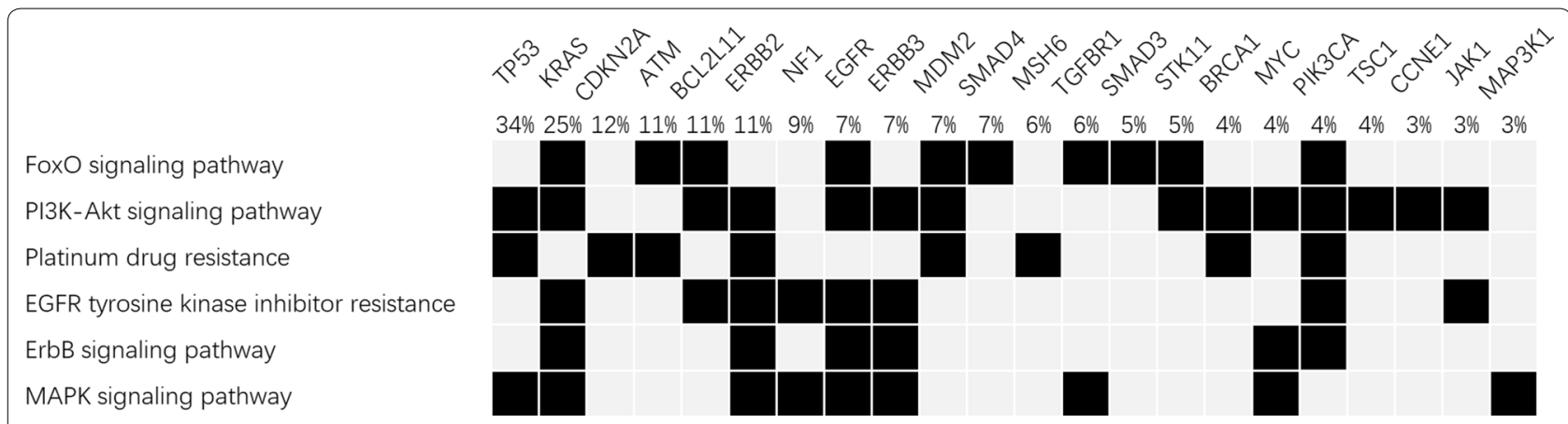

Fig. 2 Enriched pathways
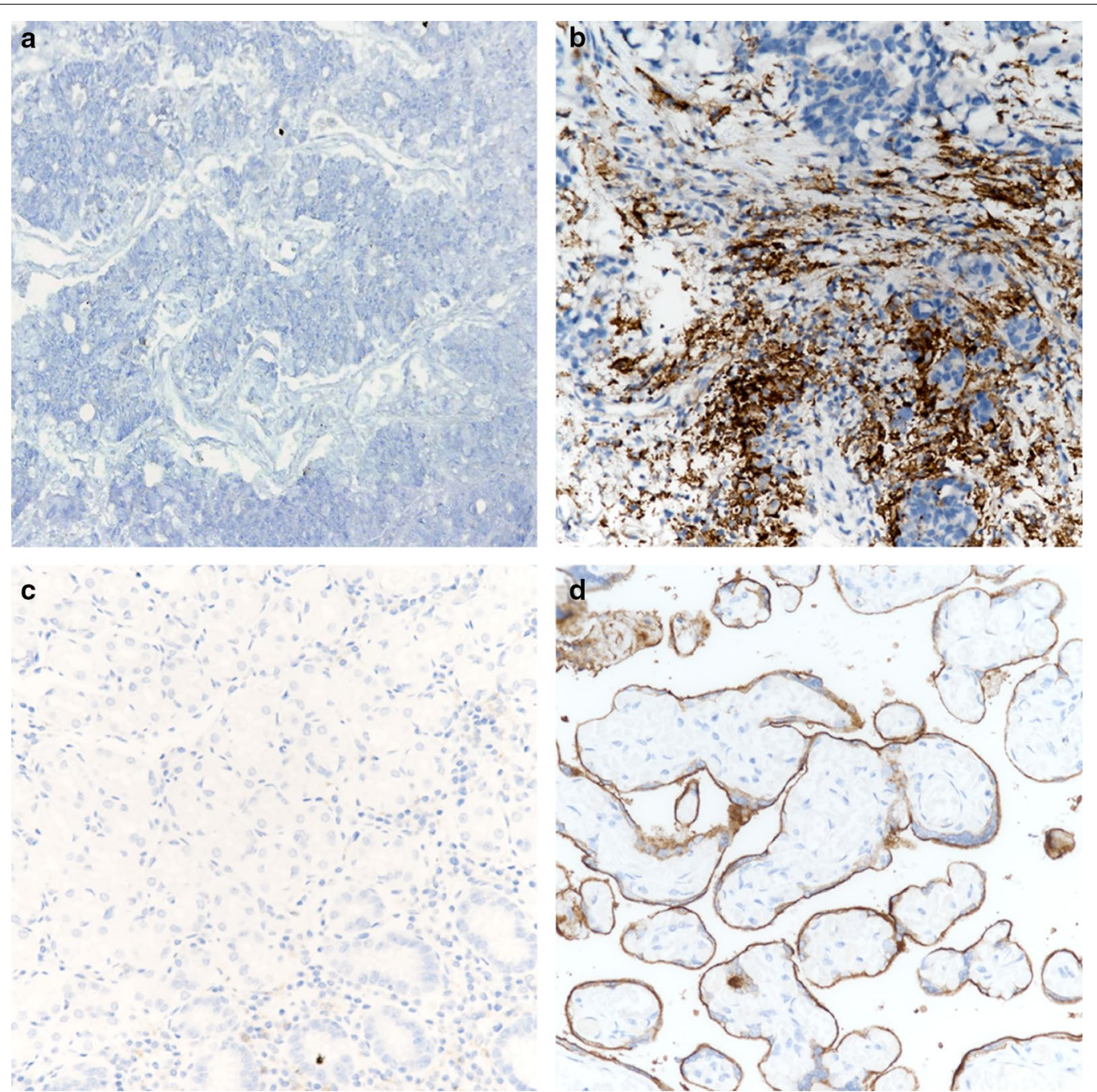

Fig. 3 The immunohistochemical results for PD-L1 were negative in patient sample A (a) and positive (1\%) in patient sample B (b). Negative control (c) and positive control (d) 


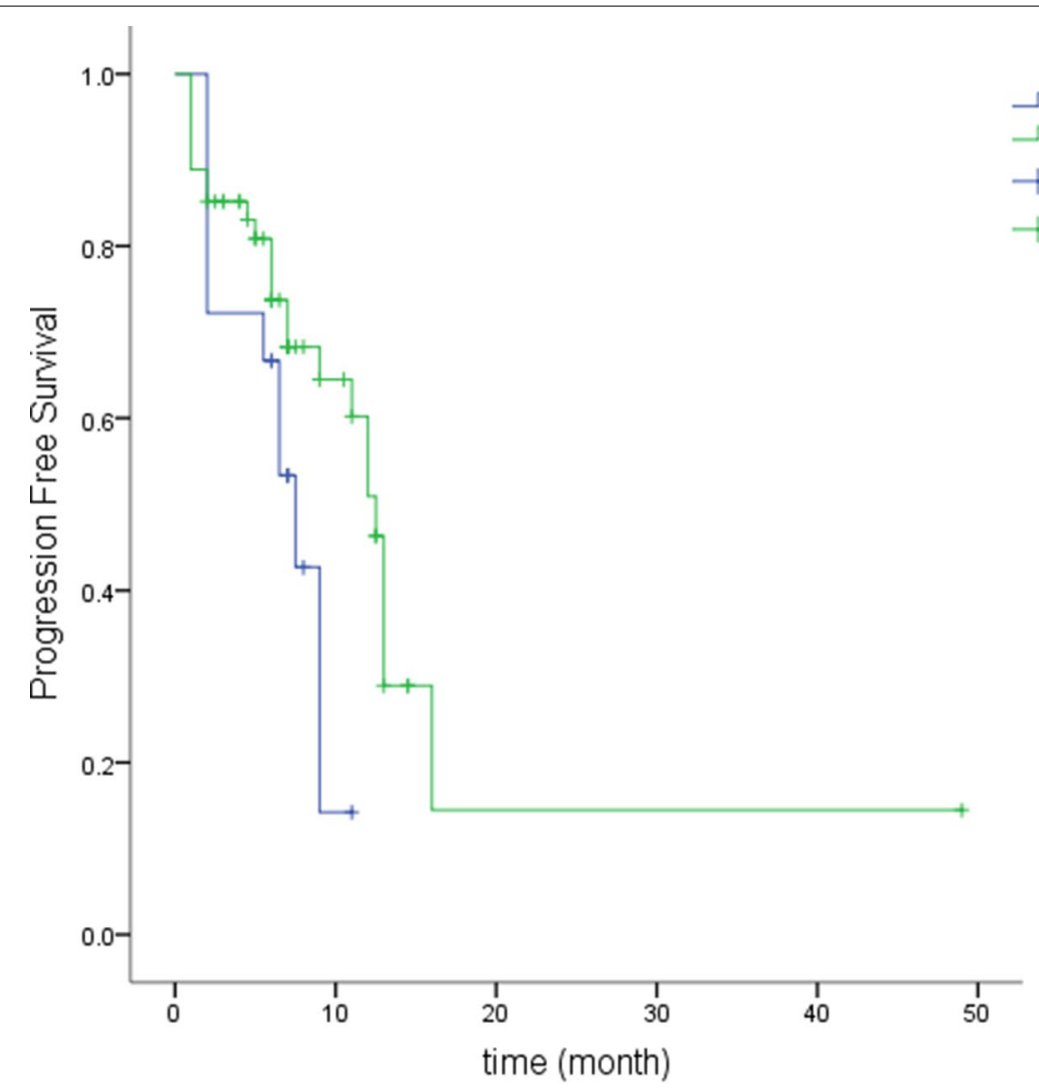

Fig. 4 PFS was 15.4 months in the bevacizumab group and 6.7 months in the chemotherapy group $(P=0.04)$

identified as commonly occurring genetic alterations, similar to the results of the present study (Table 4).

Genomic alterations, mostly rearrangements, in FGFR2 are commonly reported in cholangiocarcinoma patients in Western countries [14, 15], and these alterations result in constitutive activation of the FGFR2 receptor. In this study, a much lower FGFR2 alteration rate (2\%) was detected in the Chinese population compared with that in other populations. Patients with FGFR fusion could benefit from BGJ398 and derazantinib treatment, and prospective clinical trials are evaluating the efficacy of multiple anti-FGFR treatments on cholangiocarcinoma (NCT04093362/NCT02924376/NCT03773302/ NCT $04238715 / \mathrm{NCT} 02150967$ / NCT 03230318 / NCT03656536/NCT04088188).

ERBB2 amplifications are relatively rare in ICC compared with other types of biliary tract cancer (BTC) [16]. Due to low mortality and the number of patients with specific ERBB2 amplifications, a previous clinical trial (NCT00478140) was halted. The frequency of ERBB2 amplification in this study was $8 \%$, which is higher than that previously reported by other studies ${ }^{16}$. Notably, the disease control rate among eight patients who received anti-ERBB2 treatment with trastuzumab reached $7 / 8$, and one patient achieved a clinically resectable status. The therapeutic efficacy of anti-ERBB2 agents on ERBB2-amplified ICCs has not been previously reported, but phase II trials in China and South Korea are set to evaluate the combination of gemcitabine-based chemotherapy with trastuzumab in cases of ERBB2-amplified extrahepatic cholangiocarcinoma and gallbladder cancer (NCT02836847) and BTC (NCT03613168). Basket and multicenter trials may evaluate this treatment more adequately.

Cholangiocarcinoma patients with BRCA1/2 mutations treated with PARP inhibitors exhibited a favorable response [17]. The predictive features of germline and somatic mutations remain to be elucidated, and the germline versus somatic mutation ratio in this study was 2:12. In this study, only four patients accepted olaparib treatment, mostly due to economic factors. A recent study demonstrated that the accumulation of 2-hydroxyglutarate in association with isocitrate dehydrogenase (IDH) mutations can suppress homologous recombination and thereby induce sensitivity to PARP inhibitors [18]. These findings provided the foundation for a trial exploring the antitumoral activity of olaparib in solid tumors that harbor IDH1/2 mutations (NCT03212274). 


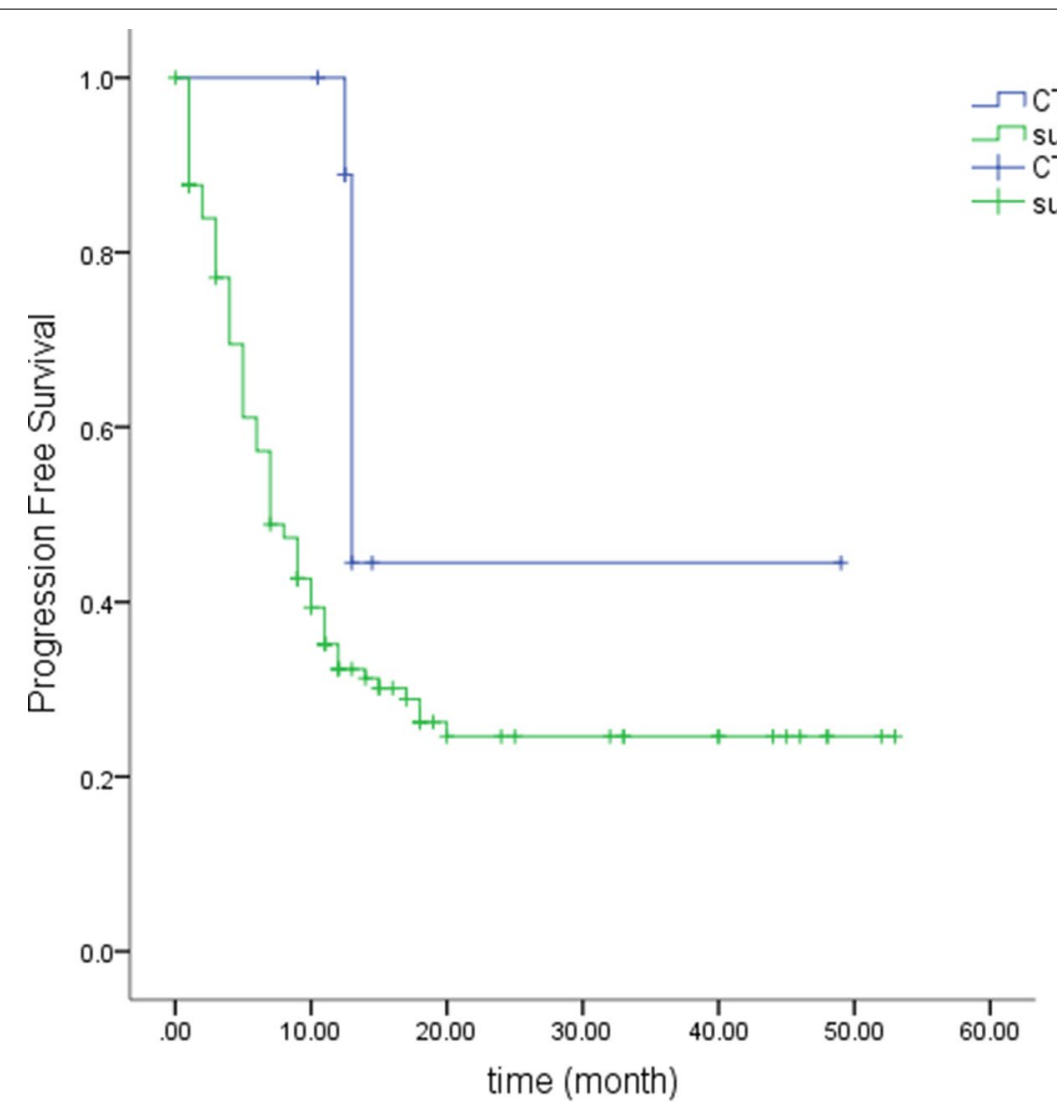




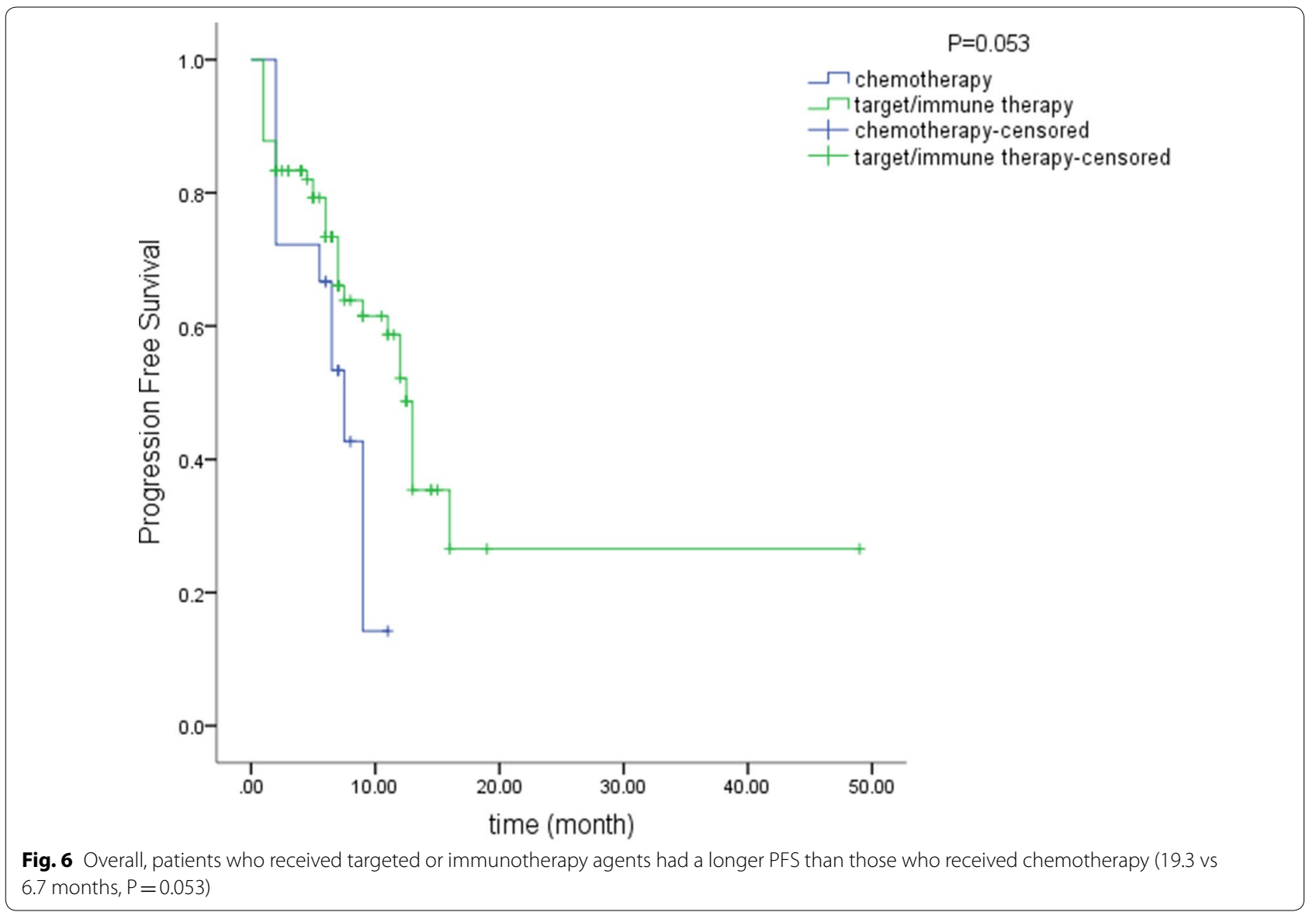

Table 4 Most commonly altered genes in intrahepatic cholangiocarcinoma

\begin{tabular}{lllll}
\hline & $\begin{array}{l}\text { Lowery } \\
\text { et al. [14] } \\
(\mathbf{N}=\mathbf{1 5 8})\end{array}$ & $\begin{array}{l}\text { Churi } \\
\text { et al. [37] } \\
(\mathbf{N}=\mathbf{5 5})\end{array}$ & $\begin{array}{l}\text { Ross } \\
\text { et al. [38] } \\
\mathbf{( N = 2 8 )}\end{array}$ & $\begin{array}{l}\text { Zou } \\
\text { et al. [39] } \\
\mathbf{( N = 1 0 2 )}\end{array}$ \\
\hline $\begin{array}{c}\text { TP53 (34\%), } \\
(\%)\end{array}$ & 20 & 29 & 36 & 38 \\
$\begin{array}{c}\text { KRAS (25\%), } \\
(\%)\end{array}$ & 7 & 24 & 11 & 17 \\
$\begin{array}{c}\text { ARID1A (17\%), } \\
(\%)\end{array}$ & 23 & 20 & 36 & 7 \\
\hline
\end{tabular}

significantly better than those of patients in the chemotherapy group. Moreover, two of the patients exhibited complete responses, and 10 patients underwent hepatectomy after systemic therapy. However, the evidence for adding bevacizumab to gemcitabine-based first-line chemotherapy is still insufficient and comparatively weak. Zhu et al. assessed the efficacy of bevacizumab in combination with GEMOX in 35 patients with advanced BTCs, and the reported PFS rate at 6 months was 63\%, which was satisfactory but below the targeted rate of $70 \%$
[23]. Iyer et al. explored the efficacy of bevacizumab combined with gemcitabine-capecitabine in 50 patients with advanced BTCs, reporting a PFS time of 8.1 months, an OS time of 10.2 months and a clinical benefit rate of $72 \%$ [24]. However, concluding that the addition of bevacizumab improved outcomes was not possible based on the results. A controlled clinical trial reported longer PFS times in the bevacizumab combination group (6.48 months) than in the GEMOX group (3.72 months) [25]. There are cases of ICCs being successfully treated with bevacizumab combined with oxaliplatin-based chemotherapies as a consequence of misdiagnosed colorectal carcinoma liver metastasis [26, 27]. Evidence supporting neoadjuvant or conversional treatment in ICC is lacking, but our results showed that late staged patients accepted combination of chemotherapy and bevacizumab treatment and consecutive operation had a longer PFS time than those underwent radical surgery. Patients may benefit from pre-operation systemic treatment. Thus, bevacizumab is an underutilized target agent for ICCs, and its efficacy and safety should be further evaluated.

The reported frequency of MSI-H in cholangiocarcinoma patients ranges widely from $1 \%$ to $10 \%[28$, 
29], and a prospective study reported an MSI-H rate of $0.5 \%$ in unselected cholangiocarcinoma patients [14]. In this study, the MSI-H rate was $2 \%$ in ICC patients; both patients accepted immunotherapy with pembrolizumab, and the therapeutic efficacy was satisfactory. The FDA approved pembrolizumab for the treatment of MSI-H/mismatch repair deficient (dMMR) tumors, which showed a predictive response to immunotherapy with PD-1 checkpoint inhibitors [30]. The KEYNOTE16 phase II study reported a $100 \%$ disease control rate for 4 MSI-H cholangiocarcinoma patients who received pembrolizumab [6], and ongoing clinical trials are accessing immunotherapy in combination with chemotherapy (NCT03111732) or radiotherapy (NCT03898895) for the treatment of cholangiocarcinoma. Additionally, a trial has been designed that combines a PD-1 antibody, a tyrosine kinase inhibitor (TKI, lenvatinib) and GEMOX for the treatment of ICCs (NCT0395197). All studies accessing the therapeutic efficacies of TKIs (including the multitarget TKIs cabozantinib [31], vandetanib [32], sorafenib [33]; the panErbB family receptor TKI afatinib [34]; the VEGF family receptor TKI cediranib [35]; and the combination of pazopanib and trametinib [36]) failed to show survival improvements. The use of checkpoint inhibitor and TKIs in combination may bring hope to patients with ICC.

This study was retrospective, which limits its evidence grade. However, we herein integrated the genetic profiles of ICC patients in the Chinese population. To our knowledge, this is the largest study on the use of NGS results as a reference for selecting target/immune therapies.

\section{Conclusions}

The therapeutic efficacies of personalized treatments are encouraging. Notably, anti-VEGF therapy showed promising improvements in regards to tumor response and patient survival rates. In summary, patients can benefit from NGS customized therapy, and further investigations are crucial for future strategies.

\section{Supplementary information}

Supplementary information accompanies this paper at https://doi. org/10.1186/s12967-020-02437-2.

Additional file 1: Table S1. Gene list of the 417-gene panel.

Additional file 2: Table S2. Therapeutic strategies.

Additional file 3: Figure S1. Portal vein tumor thrombosis (red arrows) and tumor (green arrow) shrinkage during treatment.

Additional file 4: Figure S2. Progression-free survival was 9.0 months in the cetuximab group and 6.7 months in the chemotherapy group $(P=0.04)$.
Additional file 5: Figure $\mathbf{S 3}$. In the first 5 months of cetuximab treatment, tumor size decreased (A and B). After acquiring an NRAS mutation, the patient's tumor size increased (C).

Additional file 6: Figure S4. Tumor shrinkage after 4 weeks (2 cycles) of treatment of GEMOX and bevacizumab (red arrow). Green arrow points the gallbladder.

\section{Abbreviations}

BTC: Biliary tract cancer; CTCAE: Common Terminology Criteria for Adverse Events; dMMR: MSI-H/mismatch repair deficient; FDR: False discovery rate; FFPE: Formalin-fixed, paraffin-embedded; FUSCC: Fudan University Shanghai Cancer Center; gDNA: Genomic DNA; GEMOX: Gemcitabine and oxaliplatin; HBV: Hepatitis B virus; HCV: Hepatitis C virus; HGVS: Human Genome Variation Society; ICC: Intrahepatic cholangiocarcinoma; ICs: Immune cells; IDH: Isocitrate dehydrogenase; MSI: Microsatellite instability; MSS: Microsatellite stable; NCCR: National Central Cancer Registry of China; NGS: Next-generation sequencing; OS: Overall survival; PARP: Poly ADP-ribose polymerase; PCR: Polymerase chain reaction; PD-L1: Programmed death ligand 1; PFS: Progressionfree survival; RECIST: Response Evaluation Criteria in Solid Tumors; SNVs: Single-nucleotide variation; TCs: tumor cells; TKI: Tyrosine kinase inhibitor; TMB: Tumor mutational burden; VAF: Variant allele frequency.

\section{Acknowledgements}

Not applicable.

\section{Authors' contributions}

$L W, H Z$ and $Y Z$ contributed equally to this study. All authors had access to the study data. All authors read and approved the final manuscript.

\section{Funding}

This work was jointly supported by Wu Jieping Medical Foundation (320.2710.1857), National Natural Science Foundation of China (81874182, 81874056), National Key Project of China (2017ZX10203204-007-004).

\section{Availability of data and materials}

The datasets used and analysed during the current study are available from the corresponding author on reasonable request.

\section{Ethics approval and consent to participate}

The study protocol was approved by the FUSCC ethics committee (No. 2181611 and No. 050432-4-1911D). Written informed consent for tumor profiling was obtained from each patient upon their first admission to FUSCC.

\section{Consent for publication}

Written informed consent for publication was obtained from each patient upon their first admission to FUSCC.

\section{Competing interests}

The authors declare that they have no competing interests.

\section{Author details}

${ }^{1}$ Liver Surgery Department, Shanghai Cancer Center, Fudan University, No. 270 Dongan Rd., Shanghai 200032, People's Republic of China. ${ }^{2}$ Shanghai Cancer Center, Fudan University, Shanghai, People's Republic of China.

Received: 3 April 2020 Accepted: 24 June 2020

Published online: 06 July 2020

\section{References}

1. Chen W, Zheng R, Baade PD, Zhang S, Zeng H, Bray F, et al. Cancer statistics in China, 2015. CA Cancer J Clin. 2016;66:115-32. 
2. Jusakul A, Cutcutache I, Yong CH, Lim JQ, Huang MN, Padmanabhan N, et al. Whole-genome and epigenomic landscapes of etiologically distinct subtypes of cholangiocarcinoma. Cancer Discov. 2017;7:1116-35.

3. Razumilava N, Gores GJ. Cholangiocarcinoma. Lancet. 2014;383:2168-79.

4. Khan SA, Taylor-Robinson SD, Toledano MB, Beck A, Elliott P, Thomas $\mathrm{HC}$. Changing international trends in mortality rates for liver, biliary and pancreatic tumours. J Hepatol. 2002;37:806-13.

5. Zhang H, Yang T, Wu M, Shen F. Intrahepatic cholangiocarcinoma: epidemiology, risk factors, diagnosis and surgical management. Cancer Lett. 2016;379:198-205.

6. Le DT, Durham JN, Smith KN, Wang H, Bartlett BR, Aulakh LK, et al. Mismatch repair deficiency predicts response of solid tumors to PD-1 blockade. Science. 2017:357:409-13.

7. Abou-Alfa GK, Sahai V, Hollebecque A, Vaccaro G, Melisi D, Al-Rajabi R, et al. Pemigatinib for previously treated, locally advanced or metastatic cholangiocarcinoma: a multicentre, open-label, phase 2 study. Lancet Oncol. 2020;21:671-84.

8. Friedman AA, Letai A, Fisher DE, Flaherty KT. Precision medicine for cancer with next-generation functional diagnostics. Nat Rev Cancer. 2015;15:747-56.

9. Chen S, Zhou Y, Chen Y, Gu J. Fastp: an ultra-fast all-in-one FASTQ preprocessor. Bioinformatics. 2018;34:1884-90.

10. Li H, Durbin R. Fast and accurate short read alignment with BurrowsWheeler transform. Bioinformatics. 2009;25:1754-60.

11. Chen S, Zhou Y, Chen Y, Huang T, Liao W, Xu Y, et al. Gencore: an efficient tool to generate consensus reads for error suppressing and duplicate removing of NGS data. BMC Bioinform. 2019;20:606.

12. Li H, Handsaker B, Wysoker A, Fennell T, Ruan J, Homer N, et al. The sequence alignment/map format and SAMtools. Bioinformatics. 2009;25:2078-9.

13. Wang K, Li M, Hakonarson H. ANNOVAR: functional annotation of genetic variants from high-throughput sequencing data. Nucleic Acids Res. 2010;38:e164

14. Lowery MA, Ptashkin R, Jordan E, Berger MF, Zehir A, Capanu M, et al. Comprehensive molecular profiling of intrahepatic and extrahepatic cholangiocarcinomas: potential targets for intervention. Clin Cancer Res. 2018;24:4154-61.

15. Valle JW, Lamarca A, Goyal L, Barriuso J, Zhu AX. New horizons for precision medicine in biliary tract cancers. Cancer Discov. 2017;7:943-62.

16. Galdy S, Lamarca A, McNamara MG, Hubner RA, Cella CA, Fazio N, et al. HER2/HER3 pathway in biliary tract malignancies; systematic review and meta-analysis: a potential therapeutic target? Cancer Metastasis Rev. 2017;36:141-57.

17. Golan T, Raitses-Gurevich M, Kelley RK, Bocobo AG, Borgida A, Shroff RT, et al. Overall survival and clinical characteristics of BRCA-associated cholangiocarcinoma: a multicenter retrospective study. Oncologist. 2017;22:804-10.

18. Sulkowski PL, Corso CD, Robinson ND, Scanlon S, Purshouse KR, Bai H, et al. 2-Hydroxyglutarate produced by neomorphic IDH mutations suppresses homologous recombination and induces PARP inhibitor sensitivity. Sci Transl Med. 2017;9:eaal2463.

19. Lee J, Park SH, Chang HM, Kim JS, Choi HJ, Lee MA, et al. Gemcitabine and oxaliplatin with or without erlotinib in advanced biliary-tract cancer: a multicentre, open-label, randomised, phase 3 study. Lancet Oncol. 2012;13:181-8.

20. Malka D, Cervera P, Foulon S, Trarbach T, de la Fouchardière C, Boucher E, et al. Gemcitabine and oxaliplatin with or without cetuximab in advanced biliary-tract cancer (BINGO): a randomised, open-label, noncomparative phase 2 Trial. Lancet Oncol. 2014;15:819-28.

21. Borbath I, Ceratti A, Verslype C, Demols A, Delaunoit T, Laurent S, et al. Combination of gemcitabine and cetuximab in patients with advanced cholangiocarcinoma: a phase II study of the Belgian Group of Digestive Oncology. Ann Oncol. 2013;24:2824-9.

22. Jensen $\mathrm{LH}$, Lindebjerg J, Ploen J, Hansen TF, Jakobsen A. Phase II markerdriven trial of panitumumab and chemotherapy in KRAS wild-type biliary tract cancer. Ann Oncol. 2012:23:2341-6.

23. Zhu AX, Meyerhardt JA, Blaszkowsky LS, Kambadakone AR, Muzikansky A, Zheng $\mathrm{H}$, et al. Efficacy and safety of gemcitabine, oxaliplatin, and bevacizumab in advanced biliary-tract cancers and correlation of changes in 18-fluorodeoxyglucose PET with clinical outcome: a phase 2 study. Lancet Oncol. 2010:11:48-54.
24. Iyer RV, Pokuri VK, Groman A, Ma WW, Malhotra U, lancu DM, et al. A multicenter phase II study of gemcitabine, capecitabine, and bevacizumab for locally advanced or metastatic biliary tract cancer. Am J Clin Oncol. 2018:41:649-55.

25. Bréchon M, Dior M, Dréanic J, Brieau B, Guillaumot MA, Brezault C, et al. Addition of an antiangiogenic therapy, bevacizumab, to gemcitabine plus oxaliplatin improves survival in advanced biliary tract cancers. Invest New Drugs. 2018;36:156-62.

26. Uji M, Mizuno T, Ebata T, Sugawara G, Igami T, Uehara K, et al. A case of advanced intrahepatic cholangiocarcinoma accidentally, but successfully, treated with capecitabine plus oxaliplatin (CAPOX) therapy combined with bevacizumab: a case report. Surg Case Rep. 2016;2:63.

27. Chen $X$, Zhao F, Chen $D$, Yang $P$, Zhong $W$, Xu X, et al. Successful treatment of colorectal liver metastasis harboring intrahepatic cholangiocarcinoma: a case report. Medicine. 2018;97:e13751.

28. Winkelmann R, Schneider M, Hartmann S, Schnitzbauer AA, Zeuzem $\mathrm{S}$, Peveling-Oberhag J, et al. Microsatellite instability occurs rarely in patients with cholangiocarcinoma: a retrospective study from a German tertiary care hospital. Int J Mol Sci. 2018;19:1421.

29. Silva VW, Askan G, Daniel TD, Lowery M, Klimstra DS, Abou-Alfa GK, et al. Biliary carcinomas: pathology and the role of DNA mismatch repair deficiency. Chin Clin Oncol. 2016;5:62.

30. Le DT, Uram JN, Wang H, Bartlett BR, Kemberling H, Eyring AD, et al. PD-1 Blockade in Tumors with Mismatch-Repair Deficiency. N Engl J Med. 2015;372:2509-20.

31. Goyal L, Zheng H, Yurgelun MB, Abrams TA, Allen JN, Cleary JM, et al. A phase II and biomarker study of cabozantinib in patients with advanced cholangiocarcinoma. Cancer. 2017;123:1979-88.

32. Kessler ER, Eckhardt SG, Pitts TM, Bradshaw-Pierce EL, O'byrant CL, Messersmith WA, et al. Phase I trial of vandetanib in combination with gemcitabine and capecitabine in patients with advanced solid tumors with an expanded cohort in pancreatic and biliary cancers. Invest New Drugs. 2016;34:176-83.

33. El-Khoueiry AB, Rankin CJ, Ben-Josef E, Lenz H, Gold PJ, Hamilton RD, et al. SWOG 0514: a phase II study of sorafenib in patients with unresectable or metastatic gallbladder carcinoma and cholangiocarcinoma. Invest New Drugs. 2012;30:1646-51.

34. Moehler M, Maderer A, Ehrlich A, Foerster F, Schad A, Nickolay T, et al. Safety and efficacy of afatinib as add-on to standard therapy of gemcitabine/cisplatin in chemotherapy-naive patients with advanced biliary tract cancer: an open-label, phase I trial with an extensive biomarker program. BMC Cancer. 2019;19:55.

35. Valle JW, Wasan H, Lopes A, Backen AC, Palmer DH, Morris K, et al. Cediranib or placebo in combination with cisplatin and gemcitabine chemotherapy for patients with advanced biliary tract cancer (ABC-03): a randomised phase 2 trial. Lancet Oncol. 2015;16:967-78.

36. Shroff RT, Yarchoan M, O'Connor A, Gallagher D, Zahurak ML, Rosner $\mathrm{G}$, et al. The oral VEGF receptor tyrosine kinase inhibitor pazopanib in combination with the MEK inhibitor trametinib in advanced cholangiocarcinoma. Br J Cancer. 2017;1 16:1402-7.

37. Churi CR, Shroff R, Wang Y, Rashid A, Kang HC, Weatherly J, et al. Mutation profiling in cholangiocarcinoma: prognostic and therapeutic implications. PLoS ONE. 2014:9:e115383.

38. Ross JS, Wang K, Gay L, Al-Rohil R, Rand JV, Jones DM, et al. New routes to targeted therapy of intrahepatic cholangiocarcinomas revealed by nextgeneration sequencing. Oncologist. 2014;19:235-42.

39. Zou S, Li J, Zhou H, Frech C, Jiang X, Chu JS, et al. Mutational landscape of intrahepatic cholangiocarcinoma. Nat Commun. 2014;5:5696.

\section{Publisher's Note}

Springer Nature remains neutral with regard to jurisdictional claims in published maps and institutional affiliations. 